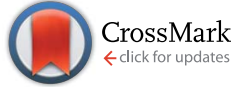

Cite this: Anal. Methods, 2015, 7, 3298

Received 11th January 2015

Accepted 27th February 2015

DOI: 10.1039/c5ay00080g

www.rsc.org/methods

\section{Using integrated absorption to calibrate optical cavity spectrometers}

\author{
D. P. Fullam, ${ }^{a}$ K. Shoji ${ }^{b}$ and D. S. Venables ${ }^{\star a}$ \\ Sensitive absorption techniques using optical cavities (such as CEAS or ICOS) generally need the \\ spectrometer response to be calibrated for quantitative measurements. Most calibrations are based on \\ the instrument response to a known, steady state absorption. Such calibrations often have drawbacks in \\ terms of cost, complexity, or convenience, especially for field measurements. In this paper, we show that \\ the relationship between the integrated absorption and a known amount of absorber provides an \\ alternative calibration strategy that yields a highly linear calibration curve and has a low uncertainty. This \\ method is straightforward to implement and offers a practical alternative to other calibration strategies.
}

\section{Introduction}

Optical cavities are an essential tool for extremely sensitive absorption measurements. ${ }^{1,2}$ Spectroscopic methods exploiting the properties of such cavities are now widely used in the laboratory and the field for quantitative measurement of gases and liquid spectra. Applications include breath analysis, ${ }^{3}$ trace gas detection, ${ }^{4,5}$ isotope ratio measurements, ${ }^{6}$ and aerosol extinction measurements. ${ }^{7,8}$ The forerunner of optical cavity methods, cavity ring-down spectroscopy (CRDS), is considered a calibration-free method as it measures the rate of decay of photons in a cavity (although calibration is sometimes recommended in practice ${ }^{9}$. Cavity-enhanced absorption spectroscopy (CEAS) and other methods measure the transmitted intensity through an optical cavity to determine the extinction coefficient, $\varepsilon:^{10}$

$$
\varepsilon=\left(\frac{I_{0}-I}{I}\right) \frac{1-R}{L}
$$

where $I$ and $I_{0}$ are the measured intensities through the sample and the reference intensity through a clean, non-absorbing matrix or vacuum. The performance of the spectrometer is determined by $L$, the length of the sample path in the cavity, and $R$, the effective mirror reflectivity. Quantitative absorption measurements require careful calibration of $R$, and of $L$ if it is not coextensive with the mirror separation. Calibration of optical cavity instruments remains a focus of recent studies. ${ }^{11-15}$

CEAS systems are generally calibrated by introducing a known, steady-state extinction into the cavity. This may be achieved using a gas of known absorption line strength at a

${ }^{a}$ Department of Chemistry and Environmental Research Institute, University College Cork, Cork, Ireland. E-mail: d.venables@ucc.ie

${ }^{b}$ Department of Chemistry, Kyoto University, Kyoto, Japan reduced pressure in an evacuable cavity. ${ }^{16}$ For systems operating at ambient pressures, or for liquid samples, a known concentration of an absorbing species is commonly used for the calibration; ${ }^{6,17,18}$ absorption by water vapour or the $\mathrm{O}_{2}-\mathrm{O}_{2}$ dimer in ambient air may also be suitable for calibrating some wavelengths. ${ }^{13}$ Equivalently, the difference in Rayleigh scattering between two gases can be used to calibrate highly sensitive spectrometers. ${ }^{19}$ Alternative approaches include using antireflection coated optics with calibrated losses, ${ }^{5}$ or measuring the phase shift of a modulated light source or the ringdown time. ${ }^{12,14,15}$ These calibration methods are often inconvenient or costly, requiring gas mixtures, multiple gas bottles, or more complex instrumentation. The requirements for these calibrations may be particularly onerous in field instruments. An alternative, robust, and accurate calibration method, applicable to the actual operating conditions of a given instrument, would be desirable.

In this paper, we demonstrate that the integrated absorption produced by a known amount of absorbing, volatile compound is an effective and accurate calibration approach for CEAS instrumentation. We relate the integrated absorption to spectrometer performance, and provide experimental evidence for the accuracy of this calibration strategy.

\section{Experimental}

A field-deployable incoherent, broadband cavity-enhanced absorption spectrometer (IBBCEAS) instrument was used to demonstrate the calibration procedure. The system was similar to other instruments and only a brief description will be given here ${ }^{20}$ The spectrometer comprised two moderately reflective $(R$ $=0.994$ to 0.998 ) dielectric mirrors with a maximum reflectivity region extending from 300 to $460 \mathrm{~nm}$. Mirrors were separated by $114 \mathrm{~cm}$; the sample inlet and outlet were $92 \mathrm{~cm}$ apart and approximately centred with respect to the cavity mirrors. This 
configuration gives rise to a dead volume between each cavity mirror and the nearest inlet or outlet. The inlet sampled laboratory air and incorporated an inline septum for introducing calibration standards. The sample was drawn through the system by a small vacuum pump and a flowmeter controlled the flowrate to between 1 and $5 \mathrm{dm}^{3} \mathrm{~min}^{-1}$. The light source of the spectrometer was a high powered LED with an emission maximum at $366 \mathrm{~nm}$. The intensity spectrum of light transmitted through the cavity was measured using an Andor Shamrock 163 spectrometer equipped with an iDUS CCD detector.

Biacetyl (butane-2,3-dione; Aldrich, 97\%) and methyl vinyl ketone (butenone; Aldrich, 99\%) were used as calibration standards. The boiling points of these compounds are $88{ }^{\circ} \mathrm{C}$ and $81.4{ }^{\circ} \mathrm{C}$, respectively, and their absorption cross sections have been reported. ${ }^{21,22}$ A $10 \mu \mathrm{L}$ microlitre syringe was used to introduce precise volumes of these liquids into the inlet flow of the system. For determining the retention time in the system, $\mathrm{NO}_{2}$ was introduced into the inlet stream from a gas-tight syringe through the septum.

\section{Results and discussion}

\section{Integrated absorption in the spectrometer}

The relationship between the integrated absorption, the amount of calibration standard in the system, and the instrument calibration parameters must account explicitly for the time-dependence of the absorber passing through the optical cavity. The instantaneous response of the system, $I(t)$, depends on the average number density, $N(t)$, and hence the number of molecules, $n(t)$, in the cavity at time, $t$ :

$$
\frac{I_{0}(t)-I(t)}{I(t)}=\frac{\Delta I(t)}{I(t)}=N(t) \frac{\sigma L}{1-R}=\frac{n(t)}{V_{\mathrm{C}}} \frac{\sigma L}{(1-R)}
$$

where $V_{\mathrm{C}}$ is the volume of the sample gas in the cavity and $\sigma$ is the absorption cross section of the molecules. The integral of the fractional intensity change, which we hereafter refer to as the integrated absorption, $A$, arising from the absorption of a single molecule is

$$
A=\int_{t=0}^{\infty} \frac{\Delta I(t)}{I(t)} \mathrm{d} t=\frac{\sigma L \tau}{V_{\mathrm{C}}(1-R)}
$$

where $\tau$ is the residence time of the molecule in the cavity. The integrated absorption, $A_{\mathrm{T}}$, from a total of $n_{\mathrm{T}}$ molecules is then

$$
A_{\mathrm{T}}=\frac{n_{\mathrm{T}} \sigma L \tau}{V_{\mathrm{C}}(1-R)}
$$

An identical integrated absorption as that in eqn (4) could be produced over the residence time by the continuous absorption of a constant number density of molecules, $N_{\mathrm{C}}$ :

$$
A_{\mathrm{T}}=\frac{\Delta I}{I} \tau=\frac{N_{\mathrm{C}} \sigma L \tau}{(1-R)}
$$

This constant number density of this time could be produced by the same total number of molecules in the flowing stream:

$$
N_{\mathrm{C}}=\frac{n_{\mathrm{T}}}{V_{\mathrm{S}}}
$$

where $V_{\mathrm{S}}$ is the volume of the sample stream giving rise to this concentration. It follows that $V_{\mathrm{S}}=f \tau$, where $f$ is the flowrate of the sample stream. Accordingly, the integrated area of a fixed number of molecules is

$$
A_{\mathrm{T}}=\left(\frac{n_{\mathrm{T}}}{f}\right)\left(\frac{L}{1-R}\right) \sigma
$$

The effective pathlength of light through the sample, $L /(1-R)$, can be determined from the slope of $A_{\mathrm{T}}$ against $n_{\mathrm{T}}$ at a particular flowrate.

\section{Calibration measurements}

The time profile of the fractional intensity change, $(\Delta I / I)$, at 366 $\mathrm{nm}$ is shown in Fig. 1 for a series of injections of biacetyl into the spectrometer inlet stream. The integrated absorption was taken as the area under the peak after removing the baseline (estimated between the start and end of the peak). The integrated intensity changed linearly with the volume of the calibration standard (Fig. 2), regardless of the flowrate. No effect associated with changes in the gas refractive index was observed, and the calculated refractive indices of the calibration gas in the spectrometer were virtually identical to that of the matrix gas. Good repeatability was found: the standard deviation of the integrated absorption of $10 \mu \mathrm{L}$ volumes of biacetyl was $3.4 \%$ ( 5 observations at $5 \mathrm{dm}^{3} \mathrm{~min}^{-1}$ ). Some of the variation is likely due to variations in the volume dispensed as the reproducibility of typical microlitre syringes is around $1 \%$.

Although eqn (7) suggests that the integrated absorption should be proportional to $1 / f$, the experimentally observed dependence indicates a more complex relationship (Fig. 3). The effect of the flowrate likely reflects the particular geometry of our sample cell: at low flowrates, greater diffusion of the sample

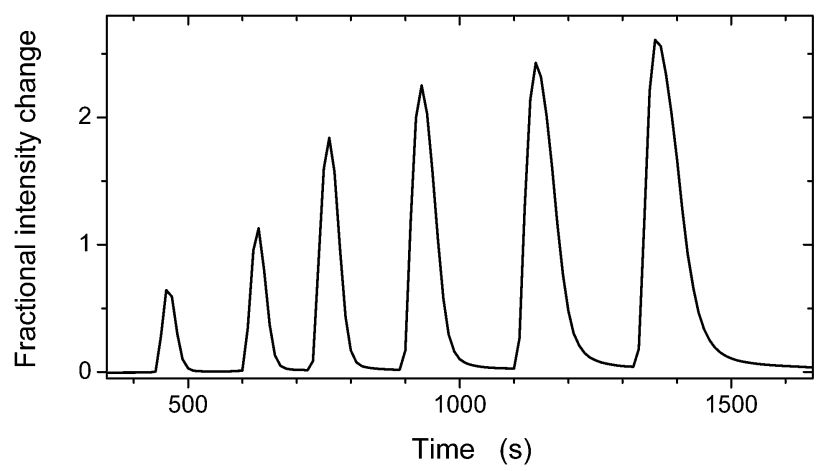

Fig. 1 Time profile of the fractional intensity change, $(\Delta / / I)$, at $366 \mathrm{~nm}$ in the spectrometer for injections of liquid biacetyl into the spectrometer inlet stream at a flowrate of $5 \mathrm{dm}^{3} \mathrm{~min}^{-1}$. The dispensed volumes (in order) were $1,2,4,6,8$, and $10 \mu \mathrm{L}$. 


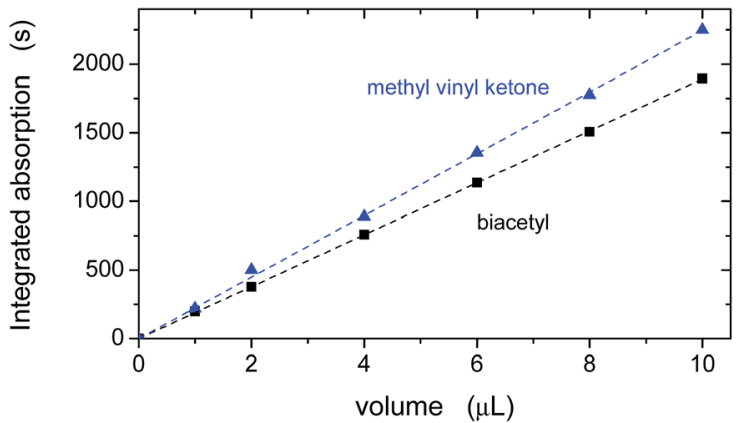

Fig. 2 Calibration curves of integrated absorption, $A_{T}$, against volume of calibration standard at a flowrate of $5 \mathrm{dm}^{3} \mathrm{~min}^{-1}$. The slopes and standard errors are: biacetyl (black squares) $189.2 \pm 0.3 \mathrm{~s} \mu \mathrm{L}^{-1}$, and methyl vinyl ketone (blue triangles) $224.6 \pm 1.6 \mathrm{~s} \mu \mathrm{L}^{-1}$. Other flowrates also produced linear calibration curves.

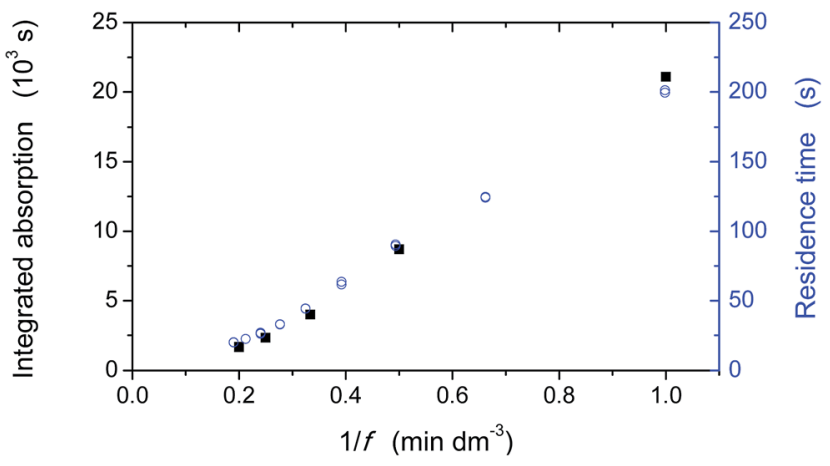

Fig. 3 Dependence on reciprocal flowrate, $1 / f$, of the integrated absorption of $10 \mu \mathrm{L}$ biacetyl (solid black squares), and of the residence time of $\mathrm{NO}_{2}$ (blue open circles) in the sample cavity. Within our control of the flowrate $( \pm 5 \%)$, both curves display the same flowrate dependence, as indicated by eqn (4).

into the dead volume near the mirrors would occur, in effect increasing the residence time and sample volume in the cavity. These spaces would be flushed more rapidly at higher flowrates. The residence time of the sample was determined with short pulses of $\mathrm{NO}_{2}$ introduced into the inlet stream (Fig. 3). The residence time displays the same dependence as the integrated intensity on the reciprocal flowrate, indicating that the enhancement in the integrated intensity is a result of the longer residence time of the sample in the cavity.

Based on the above data for $366 \mathrm{~nm}$, we calculated the characteristics for our spectrometer using the biacetyl and methyl vinyl ketone data. At $5 \mathrm{dm}^{3} \min ^{-1}, L /(1-R)$ is $156 \mathrm{~m}$, indicating the large pathlength enhancement achieved using the optical cavity. Assuming $L \approx 0.92 \mathrm{~m}$ (the distance between the sample inlet and outlet) the mirror reflectivity is estimated to be 0.9941 , in good agreement with the manufacturer's specifications. The calibration is readily applied to the full spectral range of the spectrometer.

The uncertainties associated with this calibration method are low and should be appropriate for most applications. In our results, the standard error in our calibration curves was below $1 \%$; as a result, the overall uncertainty in the calibration would be dictated by the uncertainty in the absorption cross section (typically around 5\%). Based on our repeatability results, the uncertainty of a single point calibration would be $6 \%$ and only marginally larger.

Practical use of this calibration method requires a calibration standard that meets several criteria. First, it must absorb appreciably in the spectral region of interest. Second, the standard must be sufficiently volatile to evaporate quickly in the sample stream. The standard need not be a liquid itself; it could be dissolved in a volatile solvent. Other desirable properties for the standard are chemical inertness in the spectrometer and long term stability in the storage container. Both of our calibration standards meet these criteria: the long wavelength limit of methyl vinyl ketone absorption extends to about $380 \mathrm{~nm}$ while biacetyl absorbs up to $460 \mathrm{~nm}$, allowing coverage of a significant portion of the visible spectrum. Volatile, absorbing compounds are uncommon at longer wavelengths, but $\mathrm{Br}_{2}$ or $\mathrm{I}_{2}$ (in solution) may be suitable for calibrating some spectrometers across the visible region, although these compounds have drawbacks of being corrosive and toxic.

More generally, however, calibration standards need not be volatile liquids. The central point of this work is that the integrated absorption from a known amount of any calibration standard can be used to calibrate a spectrometer, eqn (7). This applies even if the standard's concentration in the cavity and hence its absorption are time-dependent (as in Fig. 1). A calibration using the integrated absorption has the advantage of allowing a variety of other calibration standards and strategies to be used, as appropriate or convenient for a given instrument. These strategies include dispensing a fixed volume of vapour from the headspace of a volatile substance or in situ formation of the calibration standard when the stoichiometry of product formation is well understood. The method can also be used to calibrate the spectrometer response at a single wavelength; the unquantified absorption of a broad absorber can then the scaled to calibrate the overall spectral response of the system. ${ }^{23}$ Moreover, this approach is applicable to all spectral regions. An advantage of the approach is that the calibration applies directly to the particular operating conditions of the spectrometer.

\section{Conclusions}

The integrated absorption can be used to calibrate the performance of an optical cavity spectrometer when the total amount of absorber in the sample is known. We have developed the relationship between the optical cavity calibration parameters and the amount of calibration standard; the linearity of this relationship was experimentally demonstrated by adding a volatile absorbing compound in the inlet stream. The uncertainties associated with this calibration method are low and depend mainly on the uncertainty in the absorption cross section. These results show the value and potential convenience of this calibration strategy for optical cavity spectrometers, particularly when deployed outside of laboratory environments. 


\section{Acknowledgements}

We thank Science Foundation Ireland for supporting this work through grants 11/RFP/GEO3200 and 13/ISCA/2846.

\section{Notes and references}

1 K. K. Lehmann, G. Berden and R. Engeln, in Cavity RingDown Spectroscopy: Techniques and Applications, ed. G. Berden and R. Engeln, Wiley, Chichester, 2009, pp. 1-26.

2 D. Romanini, I. Ventrillard, G. Méjean, J. Morville and E. Kerstel, in Cavity-Enhanced Spectroscopy and Sensing, ed. G. Gagliaradi and H.-P. Loock, Springer, Heidelberg, 2014, pp. 1-60.

3 I. Ventrillard-Courtillot, T. Gonthiez, C. Clerici and D. Romanini, J. Biomed. Opt., 2009, 14, 064026.

4 H. Fuchs, S. M. Ball, B. Bohn, T. Brauers, R. C. Cohen, H.-P. Dorn, W. P. Dubé, J. L. Fry, R. Häseler, U. Heitmann, R. L. Jones, J. Kleffman, T. F. Mentel, P. Müsgen, F. Rohrer, A. W. Rollins, A. A. Ruth, A. Kiendler-Scharr, E. Schlosser, A. J. L. Shillings, R. Tillmann, R. M. Varma, D. S. Venables, G. Villena Tapia, A. Wahner, R. Wegener, P. J. Wooldridge and S. S. Brown, Atmos. Meas. Tech., 2010, 21-37.

5 H.-P. Dorn, R. L. Apodaca, S. M. Ball, T. Brauers, S. S. Brown, J. N. Crowley, W. P. Dubé, H. Fuchs, R. Häseler, U. Heitmann, R. L. Jones, A. Kiendler-Scharr, I. Labazan, J. M. Langridge, J. Meinen, T. F. Mentel, U. Platt, D. Pöhler, F. Rohrer, A. A. Ruth, E. Schlosser, G. Schuster, A. J. L. Shillings, W. R. Simpson, J. Thieser, R. Tillmann, R. Varma, D. S. Venables and A. Wahner, Atmos. Meas. Tech., 2013, 6, 1111-1140.

6 D. S. Sayres, E. J. Moyer, T. F. Hanisco, J. M. St. Clair, F. N. Keutsch, A. O'Brien, N. T. Allen, L. Lapson, J. N. Demusz, M. Rivero, T. Martin, M. Greenberg, C. Tuozzolo, G. S. Engel, J. H. Kroll, J. B. Paul and J. G. Anderson, Rev. Sci. Instrum., 2009, 80, 044102.

7 J. E. Thompson and H. D. Spangler, Appl. Opt., 2006, 45, 2465-2473.
8 R. M. Varma, S. M. Ball, T. Brauers, H. Dorn, U. Heitmann, R. L. Jones, U. Platt, D. Pöhler, A. A. Ruth and D. S. Venables, Atmos. Meas. Tech., 2013, 6, 3115-3130.

9 M. Schmidt, K. Maseyk, C. Lett, P. Biron, P. Richard, T. Bariac and U. Seibt, Rapid Commun. Mass Spectrom., 2010, 24, 3553-3561.

10 S. E. Fiedler, A. Hese and A. A. Ruth, Chem. Phys. Lett., 2003, 371, 284-294.

11 J. Rambo, C.-T. Lai, J. Farlin, M. Schroeder and K. Bible, J. Atmos. Ocean. Tech., 2011, 28, 1448-1457.

12 T. Laurila, I. S. Burns, J. Hult, J. H. Miller and C. F. Kaminski, Appl. Phys. B, 2010, 102, 271-278.

13 R. Thalman and R. Volkamer, Atmos. Meas. Tech., 2010, 3, 1797-1814.

14 J. Meinen, J. Thieser, U. Platt and T. Leisner, Atmos. Chem. Phys., 2010, 10, 3901-3914.

15 V. Motto-Ros, M. Durand and J. Morville, Appl. Phys. B, 2008, 91, 203-211.

16 M. Staak, E. W. Gash, D. S. Venables and A. A. Ruth, J. Mol. Spectrosc., 2005, 229, 115-121.

17 J. M. Langridge, T. Laurila, R. S. Watt, R. L. Jones, C. F. Kaminski and J. Hult, Opt. Express, 2008, 16, 1017810188.

18 Z. Qu, J. Engstrom, D. Wong, M. Islam and C. F. Kaminski, Analyst, 2013, 138, 6372-6379.

19 R. A. Washenfelder, A. O. Langford, H. Fuchs and S. S. Brown, Atmos. Chem. Phys., 2008, 8, 7779-7793.

20 J. Chen and D. S. Venables, Atmos. Meas. Tech., 2011, 4, 425436.

21 A. Horowitz, R. Meller and G. K. Moortgat, Photochem. Photobiol., 2001, 146, 19-27.

22 T. Gierczak, J. B. Burkholder, R. K. Talukdar, A. Mehouki, S. B. Barone and A. R. Ravishankara, J. Photochem. Photobiol., A, 1997, 110, 1-10.

23 J. Chen, J. C. Wenger and D. S. Venables, J. Phys. Chem. A, 2011, 115, 12235-12242. 